\title{
La prise en considération d'un événement contraire dans l'analyse de l'opposition entre l'indicatif et le subjonctif en français
}

\author{
Igor, Dreer \\ Université Ben Gourion du Néguev \\ dreer@post.bgu.ac.il
}

\section{Introduction}

Le subjonctif français est l'objet d'un nombre considérable d'études qui représentent des approches théoriques très diverses. La plupart des recherches dressent les listes des fonctions qu'exercent l'indicatif et surtout le subjonctif et des contextes linguistiques qui gouvernent l'emploi des deux modes. Parmi ces recherches, nous nommerons celles de Börjeson (1966), de Boysen (1971), de Cohen (1965), de Grevisse et Goosse (2001), de Nordahl (1969), de Togeby (1982). Les exemples montrent, pourtant, que (a) les fonctions, attribuées à chaque mode, sont soit imprécises et comme telles illimitées, soit coïncidentes et (b) les contextes syntaxiques ne gouvernent pas toujours les modes prévus. Dans ces conditions, l'intérêt des linguistes ne s'attache pas au classement des fonctions ou des emplois des deux modes, mais à la recherche d'une valeur unique qui justifierait toutes leurs significations discursives. Dans cette étude, nous proposons une hypothèse unitaire sur l'opposition entre l'indicatif et le subjonctif qui vise à expliquer la totalité de leurs emplois, y compris les emplois exceptionnels.

\subsection{Théories classiques ${ }^{1}$}

Étant donné le grand nombre de théories relatives au subjonctif, nous n'avons pas cru nécessaire d'essayer de les mentionner toutes dans cet article. En revanche, nous présentons brièvement des études plus récentes qui permettent au lecteur de se faire une idée des présupposés principaux.

Rares sont les théories pour lesquelles le subjonctif est privé de signification. Poplack (1992: 242, 243), selon qui la variabilité est inhérente au subjonctif, affirme que celui-ci avec l'indicatif et le conditionnel représentent les trois variantes d'une même variable linguistique, employée dans la subordonnée sans différences de signification. D'autres approches, comme celle de Lalaire (1998), effectuée dans le cadre de la grammaire générative, attribuent le choix des modes au problème du gouvernement de la subordonnée par la principale. D'après ce point de vue, des composantes syntaxiques, lexicales ou actancielles de la principale imposent, déterminent ou gouvernent celles de la subordonnée. A la différence du modèle génératif, les fonctionnalistes, comme Touratier (1996), proposent une hiérarchie de fonctions dont dépend le choix des modes et qui détermine si le mode de la subordonnée a un contenu sémantique ou non. Autrement dit, les fonctionnalistes distinguent entre le subjonctif qui résulte du libre choix du locuteur et celui imposé comme une servitude grammaticale. Dans une perspective quelque peu différente, Gosselin (2010: 435) attribue le choix des modes à leur compatibilité avec différentes modalités : «l'indicatif n'est compatible qu'avec une modalité $[\ldots]$ de force neutre ou orientée positivement; tandis que le subjonctif fonctionne avec les autres modalités [...] à condition que la force de ces dernières soit orientée de façon strictement négative ».

Pourtant, il y a des linguistes pour qui non seulement tous les emplois du subjonctif sont significatifs, mais qui cherchent à trouver un dénominateur commun à la totalité de ses emplois. Nous nous reporterons à Nølke $(1993,2001)$ qui avance une hypothèse, liée au concept pragmatique de polyphonie. Cette théorie considère un énoncé à travers le prisme de tous les sujets potentiels qui y sont présents d'une manière implicite ou explicite. Au sein d'un énoncé, Nølke (1993: 195) distingue entre des locuteurs responsables de l'acte d'énonciation et des énonciateurs, auteurs des actes de paroles («illocutoires, 
présuppositionnels, argumentatifs, etc. ») qui constituent le sens de l'énoncé. D’une façon générale, si le locuteur s'identifie à l'énonciateur, c'est-à-dire si le locuteur prend en charge tant le contenu de la principale que celui de la subordonnée, cela implique une nuance d'objectivité en entraînant l'indicatif. $\mathrm{Si}$, au contraire, le locuteur s'identifie à des énonciateurs différents ou s'il y a deux locuteurs différents au sein d'un même énoncé (l'un étant responsable de l'énonciation et l'autre de la présupposition), cela implique une nuance de subjectivité en entraînant le subjonctif. Selon Nølke (1993: 200), le subjonctif est donc un marqueur syntaxique de polyphonie interne au sens strict.

Martin (1983 : 104, 1987 : 15-17) propose de résoudre le problème du subjonctif en recourant aux concepts logiques du «monde possible» et de l'«univers de croyance». Celui-ci est défini comme «l'ensemble des propositions que le locuteur [...] tient pour vraies» (Martin 1983 : 114). L'univers de croyance réunit l'ensemble des mondes possibles, perçus comme tels par le locuteur, et s'oppose à ce que Martin (1983 : 114) définit comme «anti-univers » qui désigne « un ensemble de propositions que le locuteur tient pour fausses, mais qui ne le sont pas nécessairement, c'est-à-dire qui pouvaient être vraies ou que l'on imagine comme telles ». Pour Martin, le subjonctif est employé lorsqu'un énoncé suppose une proposition dont la valeur de vérité est suspendue. Cela arrive lorsqu'une proposition appartient aux mondes possibles, c'est-à-dire apparaît comme possiblement vraie ou possiblement fausse dans "le monde de ce qui est » (Martin 1987: 16). Dans ce cas, il s'agit de l'emploi du subjonctif dans les contextes de doute, de volonté, de nécessité ou dans les propositions relatives. La valeur de vérité est aussi suspendue lorsqu'une proposition appartient à l'anti-univers ce qui sert à expliquer l'emploi du subjonctif dans les contextes de négation, de concession ou de jugement de valeur.

Les justifications polyphoniques ou logiques de l'emploi du subjonctif sont, à notre avis, étrangères à la valeur proprement linguistique de ce mode parce qu'elles se basent sur des concepts linguistiquement non pertinents. La preuve psycholinguistique (cf. Kirsner $2002: 358$ ) de ces concepts n'est pas claire dans le sens que leur introduction n'est pas motivée par l'emploi des formes linguistiques, mais plutôt préétablie par les linguistes. En plus, ces approches soit ne réussissent pas à rendre compte de certains emplois du subjonctif (c'est notamment le cas de l'emploi factuel), soit leur explication semble assez peu convaincante en raison de son caractère pas assez abstrait pour embrasser les emplois les plus divers du subjonctif.

Avec toute la différence qui existe entre les analyses de Lagerqvist (2009) et Soutet (2000), les deux linguistes présentent le subjonctif, par opposition à l'indicatif, comme suspendant d'une manière générale la valeur assertive de l'énoncé. Pour Lagerqvist, l'indicatif exprime une essence (un contenu sémantique) actuelle, tandis que le subjonctif exprime le non actuel ou une essence virtuelle. Autrement dit, ce dernier a la capacité de virtualiser l'action. Pour Soutet, le subjonctif signifie, à la différence de l'indicatif, que la vérité de proposition n'est pas prise en charge par le locuteur ou par un énonciateur (celui qui assume l'énoncé). Pourtant, sans parler de certaines notions de l'approche polyphonique que les deux linguistes manient, leur argumentation n'est pas toujours convaincante. Lagerqvist, par exemple, pose le principe de la «surdétermination » selon lequel il est possible d'« échanger» les essences qui devraient avoir des valeurs sémantiques uniques de telle façon que l'indicatif exprimera le virtuel, et le subjonctif l'actuel. La notion «non prise en charge » que Soutet attribue au subjonctif n'est pas suffisante non plus pour expliquer tous les emplois factuels du subjonctif. Toutefois, ce qui nous semble important, c'est que les deux linguistes cherchent à expliquer les emplois de l'indicatif et du subjonctif dans différents environnements syntaxiques ainsi que l'alternance entre les deux modes par leur contenu sémantique (ou leurs signifiés).

La psychomécanique de Guillaume (1970), développée par Moignet (1959) et Wilmet (1998), est un exemple d'une théorie qui fournit une explication proprement linguistique de l'emploi du subjonctif. Guillaume propose une valeur linguistique unique du subjonctif ou plutôt même une attitude de pensée sous-jacente. Il considère le subjonctif comme une étape de l'opération de pensée (nommée " chronogénèse ») au cours de laquelle l'image-temps se crée dans l'esprit. Le subjonctif appartient à la deuxième étape, postérieure à celle de l'infinitif mais antérieure à celle de l'indicatif, et représente le mode du temps non divisible en époques passé-présent-futur, c'est-à-dire le mode du possible ou de la non-actualisation. 
Il faut reconnaitre que cette explication du subjonctif est présentée de façon très explicite. Pourtant, le fait absolument incontestable que ce mode se réfère à une vaste époque indivisée rend difficile l'explication certains emplois du subjonctif, comme ceux attestés dans les subordonnées concessives ou les subordonnées, introduites par après que, sur quoi nous reviendrons ultérieurement. On peut, certes, attribuer le subjonctif à une étape de la formation de l'image-temps, comme le fait Stefanini (1992 : 1736), afin d'expliquer ces emplois dans le cadre de l'hypothèse guillaumienne. Cependant la valeur proprement linguistique de ce mode ne désigne pas cette appartenance, comme d'ailleurs, ni la virtualisation, ni la «non prise en charge» qui pourraient être psycho-sémantiquement vraies mais communicativement non pertinentes. Pourtant, la pertinence communicative est un élément fondamental de l'analyse linguistique parce qu'elle est la plus apte à expliquer les lois des phénomènes linguistiques.

\begin{abstract}
Cela ne signifie pas la communication soit la seule fonction des langues, encore moins que les langues constituent l'unique moyen de communiquer. Cela signifie que pour déterminer ce qui est proprement linguistique parmi tous les aspects de la réalité physique du langage, on prendra en compte ce qui contribue de quelque façon à la communication, c'est-à-dire ce qui est pertinent selon le point de vue choisi, et qu'on laissera pour une part aux autres disciplines le soin d'étudier les autres aspects. (Clairis $2005: 18$ )
\end{abstract}

On ajoutera que la référence à la communication ne signifie pas l'intégration d'une composante pragmatique dans la description des modes. Contrairement à ce qu'on croit parfois, le travail du linguiste ne consiste pas à décrire les intentions des locuteurs ni le sens linguistique des énoncés (ce qu'on retrouve, par exemple, chez Ducrot 1972). Bien que « la langue [permette] d'exprimer (dans le discours) le monde et la pensée » (Gosselin 2010 : 11), elle ne se compose pas de pensées ni d'énoncés illimités, mais de signes linguistiques, et la tâche du linguiste consiste à rendre compte de l'emploi de ces signes par les locuteurs de la langue en question.

\begin{abstract}
Therefore, signals and meanings contribute to the creation of the whole [message], but do not compose it. The relationship is similar to that of the carpenter's tools and the edifice he builds. The edifice is built by means of the saws and hammers he uses. However, a house is not made up of saws and hammers, nor does the carpenter leave his saws and hammers in each house he builds. [...] And just as the investigation of tools and their strategies of use belongs to a theory of carpentry while the investigation of houses and their component parts belongs to the study of architecture, so too the investigation of signals and meanings belongs to a theory of language while the investigation of messages lies outside that theory. (Reid \& Gildin 1979: 170)
\end{abstract}

Si nous croyons qu'il ne faut pas se baser sur des considérations de nature pragmatique pour élaborer une explication des emplois de l'indicatif et du subjonctif, c'est parce que nous nous appuyons sur l'idée de Saussure (2001 [1916]), bien résumée par Benveniste (1966 : 64), qui rejette le rapport entre la langue et la pensée.

Poser ces deux termes, pensée et langue, comme solidaires et mutuellement nécessaires ne nous indique pas comment ils sont solidaires, pourquoi on les jugerait indispensables l'un à l'autre. Entre une pensée qui ne peut se matérialiser que dans la langue et une langue qui n'a d'autre fonction que de "signifier», on voudrait établir une relation spécifique, car il est évident que les termes en présence ne sont pas symétriques. [...] A strictement parler, la pensée n'est pas une matière à laquelle la langue prêterait forme $[\ldots]$

\title{
1.2 L'opposition entre l'indicatif et le subjonctif en français : nouvelle approche
}

Dans ce qui suit, nous proposons une approche unitaire qui consiste à considérer la langue comme un système de signes saussuriens qui sert à la communication entre les hommes et, comme tel, est déterminé par sa fonction communicative et par les caractéristiques de ses locuteurs (cf. Dreer 2007 : 258). Cela signifie que la pertinence communicative influence la forme de la langue et les faits linguistiques (cf. Martinet 1973 : 339). Autrement dit, seuls la forme et les faits qui répondent à des besoins communicatifs 
seront pertinents. Comme la communication humaine nécessite un ensemble de signes perceptibles, notamment une image acoustique, liée à un concept ou une valeur linguistique, nous considérons des signes saussuriens - par opposition à des mots, des phrases ou des propositions - comme unités analytiques de base. Non seulement la communication humaine n'est pas toujours produite en mots et en phrases, mais ceux-ci sont trop divers et indistincts - contrairement aux signes linguistiques - pour distinguer des idées «d'une façon claire et constante» (Saussure 2001 [1916] : 155). Et comme pour Saussure (ibid, p. 99), à un signifiant correspond un seul signifié, les valeurs linguistiques des signes justifient leur distribution dans différents contextes. Pour ce qui regarde notre étude, nous cherchons à postuler un dénominateur commun, une valeur unitaire qui soit à la base de tous les emplois, de toutes les fonctions du subjonctif et dont l'opposition avec la valeur postulée de l'indicatif explique le choix entre les deux modes partout dans la langue.

Nous visons à traiter l'indicatif et le subjonctif en opposition, le mécanisme du langage reposant, selon Saussure (ibid, p. 167), sur des oppositions entre les signifiés et les signifiants « et sur les différences phoniques et conceptuelles qu'elles impliquent». Notre hypothèse est qu'au sein de cette opposition, l'indicatif désigne une action, un devenir ou un état (désormais " événement », sauf indication contraire) se déroulant au présent, ayant eu lieu au passé ou ayant de fortes chances de se réaliser dans l'avenir. Le subjonctif indique, à l'inverse, la prise en considération implicite d'un événement contraire, c'est-à-dire un événement qui pourrait se produire contrairement à ce que le locuteur avait supposé. Aussi postulonsnous que l'indicatif signifie un événement, tandis que le subjonctif signifie un événement contraire considéré. Ces valeurs contribuent à différentes fonctions de l'indicatif et du subjonctif et impliquent la variété de significations spécifiques ou contextuelles des deux modes qui sont observables dans le discours. Par exemple, on distingue le subjonctif de volonté, le subjonctif d'hypothèse, le subjonctif de doute ou d'appréciation subjective. Cependant, les valeurs relèvent de la langue, alors que les significations n'appartiennent pas à la langue, mais à sa réalisation et, considérées comme telles, ne représentent pas des unités linguistiques. La distinction entre les valeurs linguistiques et leurs significations impliquées peut être mieux illustrée par analogie avec un instrument de musique, suggérée par Kirsner (2004: 6), selon qui ces significations ne font pas partie intégrante de l'instrument communicatif appelé langue, juste comme les fugues de Bach ne font pas partie intégrante de l'instrument de musique appelé clavecin. Les valeurs postulées sont plus abstraites que leurs significations et ne servent que d'indices dont l'interlocuteur déduit une signification impliquée. En d'autres termes, nous postulons que l'indicatif implique un événement per se, alors que le subjonctif évoque à l'interlocuteur la prise en considération d'un événement contraire.

À cela on pourrait rétorquer que l'approche en termes d'événement contraire ou plutôt de contrefactualité inhérente à tous les énoncés n'est pas nouvelle de sorte que, selon Martin, résumé en Nølke (1993: 8), " dire qu'il n'y a pas un nuage dans le ciel, c'est admettre malgré tout qu'il aurait pu en être autrement ». Ce point de vue présente néanmoins quelques inconvénients. D'abord, la contrefactualité s'applique aux énoncés qui, comme nous l'avons signalé plus haut, relèvent du monde extralinguistique, c'est-à-dire de la parole et pas de la langue. Ensuite, Martin et Nølke proposent, respectivement, les concepts préétablis de contrefactualité, de mondes possibles et de polyphonie dont l'évidence psycholinguistique reste floue. Ces concepts ne sont pas justifiés par l'emploi de formes linguistiques, mais représentent, selon Saussure (2001 [1916] : 153), « des concepts forgés par les grammairiens, et dont on ne sait s'ils correspondent réellement à des facteurs constitutifs du système de la langue ». Alors, même si, logiquement ou pragmatiquement, l'idée de contrefactualité est souvent sous-jacente, cela ne constitue pas cependant un fait communicativement pertinent.

L'opposition modale entre l'indicatif et le subjonctif peut être mieux illustrée par ce couple d'exemples contrastifs :

(1) Je ne pense pas qu'il est malade.

(2) Je ne pense pas qu'il soit malade. 
(3) Je cherche une personne qui sait le russe.

(4) Je cherche une personne qui sache le russe.

L'indicatif permet d'interpréter l'exemple en (1) au sens de "il est plutôt en bonne santé », et le subjonctif en (2) au sens de «il est peut-être en bonne santé, mais sa maladie n'est pas exclue ». L'indicatif en (3) est employé au sens de « on peut trouver un russophone ici », tandis que le subjonctif nous permet d'interpréter l'exemple en (4) comme «il n'y aucune garantie qu'on puisse trouver un russophone ici ». Ces interprétations montrent que la valeur un événement contraire considéré du subjonctif est plus complexe que celle un événement de l'indicatif étant donné que le subjonctif implique deux événements à la fois : celui qui s'est produit ou se produira («il est peut-être en bonne santé », « on peut trouver un russophone ici ») et son opposé possible (« sa maladie n'est pas exclue », « on peut ne pas trouver un russophone ici »). Cette double implication conditionne l'emploi du subjonctif pour exprimer des situations où les chances d'être et de ne pas être sont égales. Citons Guillaume (1970) à ce propos :

Le propre de la notion de possible est d'annuler la capacité d'actualité (= chances d'être) par une capacité égale et contraire (= chances de ne pas être). (Guillaume 1970: 33)

Aussi bien, les valeurs postulées permettent d'expliquer pourquoi l'indicatif sert une option fondamentale et le subjonctif est une option moins fréquente. Alors que l'usage de l'indicatif est associé avec une situation régulière ou un résultat prévisible, celui du subjonctif est associé avec l'inhabituel ou l'imprévisible. Le subjonctif laisse supposer que la communication concerne une situation différente de ce à quoi on pourrait normalement s'attendre.

\section{Les buts communicatifs de l'emploi de l'opposition indicatif/subjonctif}

L'opposition entre l'indicatif et le subjonctif sert souvent à exprimer différents buts communicatifs. Nous parlons de l'usage standardisé ou idiomatique d'une valeur linguistique en combinaison avec d'autres valeurs pour contribuer à des significations particulières dans des contextes d'apparition. La formulation de buts communicatifs reflète la tendance vers la conformité et la systématisation de l'usage de signes linguistiques qui simplifient la communication. L'opinion générale (voir Soutet 2000 : 59-61, 80-81, 8384) est que l'indicatif et le subjonctif s'imposent souvent selon que la vérité de la subordonnée est prise en charge, c'est-à-dire assumée ou non, respectivement, sauf dans les cas où le choix de l'un ou de l'autre mode est conditionné par le mécanisme de la subordination, c'est-à-dire par diverses conjonctions ou constructions. Nous proposons de distinguer au moins deux buts communicatifs que les locuteurs francophones poursuivent en choisissant entre l'indicatif et le subjonctif, indépendamment même des circonstances de leur emploi. A notre avis, cette opposition modale est employée :

(i) soit pour marquer le degré de la prise en charge d'un événement par le locuteur ;

(ii) soit pour exposer des faits avec ou sans prise en considération d'un événement contraire.

Pourtant, nous affirmons que dans les deux cas, l'emploi des modes est justifié par leurs valeurs linguistiques : l'indicatif est employé pour mettre en évidence un événement, tandis que le subjonctif est employé pour faire ressortir la prise en considération d'un événement contraire. Afin de neutraliser le rôle d'intuition dans notre analyse, nous nous appuyons sur un ensemble d'exemples attestés qui apparaissent dans des contextes qui explicitent souvent le choix entre les deux modes.

\subsection{L'opposition indicatif/subjonctif et la (non-)prise en charge d'un événement}

Globalement, il est vrai que les locuteurs francophones emploient souvent l'opposition modale entre l'indicatif et le subjonctif pour faire apparaître leur vision de la réalité d'événements. Nous postulons que l'emploi de l'indicatif implique que le locuteur prend en charge le résultat de l'événement en question, alors que celui du subjonctif témoigne que le locuteur cherche à éviter de l'assumer. Encore faut-il préciser que par prise en charge, nous entendons la responsabilité du locuteur vis-à-vis du résultat de l'événement et non vis-à-vis de la vérité d'un énoncé, comme c'est le cas le plus souvent. C'est parce que, 
strictement parlant, ni l'indicatif ni le subjonctif ne peuvent servir de critère de vérité, les deux modes n'étant qu'une représentation de la réalité que le locuteur veut communiquer à son interlocuteur. Autrement dit, comme l'écrit de Jonge (2001: 81), les événements exprimés par l'indicatif ne sont pas nécessairement factuels, mais l'effet visé sur l'interlocuteur a pour but de le persuader qu'ils le sont. Notre opinion est qu'en se servant de l'indicatif, le locuteur attire l'attention de l'interlocuteur sur un événement «qui est perçu comme existant, ayant existé ou ayant de fortes chances d'exister » (Soutet 2000 : 55). Par contre, la valeur du subjonctif permet au locuteur de se distancier d'un événement actuel, en en détournant l'attention de l'interlocuteur pour l'attirer sur un événement contraire considéré. C'est pourquoi, l'indicatif est employé afin de présenter les événements certains ou probables, alors que le subjonctif est employé pour les événements incertains ou possibles. Cette réflexion est illustrée par la série d'exemples authentiques en (5)-(9).

Dans l'exemple en (5), on trouve l'indicatif avec l'expression anticipative avoir peur qui introduit généralement le mode subjonctif. L'emploi de l'indicatif, renforcé par le contexte, met un accent sur le caractère inévitable d'un événement, au moins tel qu'il est perçu par Mme Peyrony qui croit que les boxeurs vont certainement tomber par-dessus les cordes du ring.

(5) Le public est fait de copains, de grandes sœurs, de poupons, d'amantes, sans oublier la mère, la classique Mme Peyrony qui tout à l'heure, quand le combat bousculera les garçons contre les cordes, de son côté - et elle est assise au second rang, - avancera l'épaule, étendra le bras (bravo pour la garde !), détournera la tête (moins bien), et criera avec épouvante: «J'ai peur qu'ils vont tomber!» (Montherlant 1954 : 147)

On objectera que l'indicatif apparaît en (5) parce que le verbe représente une périphrase aller + infinitif qui, selon Nordahl (1969 : 93, 97), n'est guère employée au subjonctif. Afin de lever cette objection, nous pouvons répondre que, généralement, cette périphrase implique que l'événement en question est sur le point de se produire, c'est-à-dire que le locuteur le considère comme incontestablement réel (voir Fleischman 1982) ce qui justifie la corrélation de cette périphrase avec le mode indicatif.

A l'instar de l'exemple en (5) ci-dessus, la prise en charge du résultat est attestée en (6) où le verbe souhaiter, majoritairement suivi du subjonctif, entrâne l'indicatif qui attire l'attention de l'interlocuteur sur l'espoir que l'événement désiré va se produire. Dans ce contexte, un guide, accusé de l'homicide involontaire d'un groupe d'élèves dans les Alpes, espère que les familles touchées le pardonneront un jour.

(6) En s'adressant une dernière fois au tribunal, le guide a déclaré : «A toutes les familles touchées [...], je souhaite que le temps leur permettra d'entendre ce que je vais leur dire : je leur demande pardon. » (Libération 1999 : 18)

On pourrait formuler une objection d'après laquelle le verbe souhaiter possède des significations différentes de sorte que la signification vouloir commande le subjonctif, alors que celle d'espérer régit le mode indicatif (voir Cohen $1965: 81-82$ ), comme c'est le cas en (6). Pourtant, cette explication contredit le principe d'invariance, soutenu dans cette étude et bien formulé par Guillaume dans L'architectonique du temps $(1970)^{2}$ :

La tendance du langage est d'obtenir, à partir d'une seule et même forme psychophonétique, qui en soi ne change pas, sa position mentale en système étant déterminée une fois pour toutes, la diversité d'effets de sens dont elle se montre capable sans abandon de sa nature essentielle, invariante.

Si nombreuses que puissent être les conséquences du signe linguistique, elles trouvent leur limite dans l'unité, maintenue au fond de la pensée, de sa condition. C'est comme condition une que le signe linguistique existe dans la langue ; et comme véhicule de conséquences variées qu'il se manifeste dans le langage (dans le discours). Une tâche du linguiste-grammairien est de faire apparaître l'unité sous la diversité des conséquences produites. (Guillaume 1970: 37-38, notes)

Aussi bien, le seul fait d'espérer ne garantit pas que le résultat d'un événement soit assumé par le locuteur. C'est notamment le cas de l'exemple en (7) ci-dessous où le subjonctif suit le verbe espérer. 
Pourtant, le français standard requiert l'emploi de l'indicatif dans ce contexte syntaxique vu que «l'espérance [...] ne naît que de l'accès à ce sentiment [de probabilité] » (Guillaume 1970: 38), alors que le mode subjonctif est souvent attribué à un événement désiré qui ne s'est pas encore produit, dont on ne sait pas s'il va se produire et dont le résultat n'est pas certain. C'est pourquoi, il nous semble plus judicieux d'admettre que c'est la valeur un événement contraire considéré du mode subjonctif qui justifie tous ses emplois, y compris celui en (7). Ici, le subjonctif marque un résultat incertain qui découle de deux possibilités contraires, impliquées à la fois. Cela laisse entendre que le docteur Rieux, tout en espérant que sa femme guérira, ne peut garantir sa guérison, comprenant qu'elle est sérieusement et peutêtre même incurablement malade ${ }^{3}$.

(7) Rieux répondit que sa femme se soignait hors de la ville. «Dans un sens, avait dit Grand, c'est une chance. » Le docteur répondit que c'était une chance sans doute et qu'il fallait espérer seulement que sa femme guérît. (Camus 1947 : 79)

Force est bien de reconnaître que la commande lexicale n'est pas toujours suivie d'un mode requis par la grammaire traditionnelle. Cette situation s'observe aussi en (8) où le subjonctif suit le verbe savoir qui comme porteur de factualité - doit commander le mode indicatif. Dans cet exemple, la Vouivre, une créature de contes populaires, essaie de comprendre la nature des émotions humaines. Le subjonctif implique qu'il lui est difficile de s'imaginer mortelle.

(8) Si je savais que je doive mourir, mettons dans trente ans, est-ce que tu crois que je changerais? (Aymé $1972: 238$ )

Ce changement de mode peut être attribué à un changement de modalité dans la proposition principale, notamment à «l'insertion de la modalité hypothétique » (Soutet $2000: 76$ ) qui concerne un événement irréalisable soit dans le présent, soit dans le passé. Comment expliquer alors l'emploi de l'indicatif, bien attesté dans ce contexte? Nous affirmons que ce mode n'a rien d'exceptionnel pour peu que le locuteur s'engage au résultat de l'événement en cause, sans prendre en considération un événement contraire, comme c'est représenté par (9). Tandis que l'exemple en (8) ci-dessus implique l'incertitude du personnage de la Vouivre, ici l'indicatif implique que le personnage de Martin prend en charge le contenu du savoir, sans supposer d'autres possibilités.

(9) «Est-ce que tu pourrais être heureuse d'avoir mangé, tout ce que tu aimes le mieux, petite lune, si tu savais qu'à côté de toi il y $a$ une famille qui n'a pas mangé depuis deux jours?»

Alia secouait la tête.

«Est-ce que tu pourrais être heureuse de regarder le ciel, la mer, les fleurs, ou d'écouter le chant des oiseaux, si tu savais qu'à côté de toi, dans la maison voisine, il y $a$ un enfant qui est enfermé sans raison, et qui ne peut rien voir, rien entendre, rien sentir?»

« Non », disait Alia. (Le Clézio 1978 : 197)

De même, l'explication du choix entre l'indicatif et le subjonctif qui n'est pas fondée sur leurs valeurs linguistiques, mais sur un changement de modalité nous semble peu probable lorsqu'il s'agit de l'insertion de la modalité interrogative. S'il est vrai que l'interrogation avec inversion favorise l'emploi du subjonctif, tandis que celle avec est-ce que favorise l'indicatif, on doit reconnaître aussi que le subjonctif n'en est pas exclu, bien que mal accepté. Et pourtant, cet emploi, comme d'ailleurs tous les autres emplois du subjonctif, s'analyse comme justifié par sa valeur linguistique un événement contraire considéré. C'est ce que l'on observe en (10) où Elisabeth, amoureuse d'un homme marié qui ne veut pas divorcer, lui laisse entendre que son amour est malheureux, mais s'aperçoit du manque de compréhension de sa part.

(10) - Franchement, Claude, est-ce que tu trouves que l'expérience de ce mois ait été heureuse ? dit-elle.

- Quelle expérience ? dit-il.

Le sang monta aux joues d'Elisabeth et sa voix trembla un peu.

- Si nous avons conservé nos rapports après l'explication du mois dernier, c'était à titre d'expérience, l'as-tu oublié ? 


\author{
- Ah ! oui..., dit Claude. \\ Il n'avait pas pris au sérieux l'idée d'une rupture [...] \\ - Tu es si simple au fond; tu ne t'es jamais rendu compte que je n'étais pas \\ heureuse. (Beauvoir $1943: 86,87$ )
}

Le décalage entre l'emploi de l'interrogation sans inversion dans la principale et du subjonctif dans la subordonnée tient d'une part au fait que, pragmatiquement parlant, l'interrogation exprimée par est-ce que ou par l'intonation suggère à l'interlocuteur une demande de confirmation d'un jugement qui paraît évident au locuteur. Citons Huot (1986) à ce propos :

\begin{abstract}
En disant Voulez-vous que je vous accompagne? on exprime, par politesse, une incertitude qui laisse à l'interlocuteur la liberté complète de dire oui ou non, sans que son refus éventuel soit désobligeant (ou éventuellement contraint), comme il pourrait l'être en réponse à : Vous voulez que je vous accompagne? par quoi le locuteur offre son aide en laissant entendre qu'il considère que l'interlocuteur la désire. (Huot 1986 : 102 , note 18 )
\end{abstract}

D'autre part, l'emploi du subjonctif qui, nous l'avons postulé, suggère la prise en compte d'un événement contraire permet au locuteur de se distancier de ce jugement. C'est pourquoi, le recours à ce mode laisse exprimer sinon une opposition, du moins une différence entre le jugement que le locuteur porte sur des événements présentés dans la subordonnée et le jugement qu'il croit être énoncé ou présupposé à ce propos par d'autres. C'est notamment le cas de l'exemple en (10) ci-dessus qui peut être traduit par une lourde paraphrase du type : « tu trouves vraiment que l'expérience de ce mois a été heureuse ? Parce que moi, je pense autrement ».

Malgré l'opinion générale que le subjonctif est « franchement exclu avec l'interrogation traduite par la seule mélodie ascendante de la phrase » (Soutet $2000: 8$ ), cet emploi s'observe dans l'exemple en (11) et s'analyse de la même manière que l'exemple en (10) ci-dessus. En (11), le subjonctif dans la subordonnée implique, d'une part, la demande du personnage de Charlotte de ranger la chambre et, d'autre part, le manque de disposition de la part du personnage de Léopold à le faire. Autrement dit, on pourrait paraphraser (11) comme «vous pensez vraiment que je dois y aller maintenant? Parce que moi, je voudrais remettre cela à plus tard ».

(11) Charlotte : Avez-vous rangé votre chambre ? Celie est vieille, non, pour faire un

lit ? Ou frotter un parquet. Vous devriez le faire. [...]

Léopold: Bon, bon. Je... Vous pensez que je doive y aller maintenant? La musique est si jolie... (Sagan $1962: 62,63$ )

La non-prise en charge se manifeste aussi par l'emploi du subjonctif dans les subordonnées, introduites par les verbes, les expressions et les conjonctions de volonté ou nécessité (avoir envie, désirer, exiger, souhaiter, vouloir, falloir dans l'expression il faut, etc.), de doute (douter, il est douteux, mettre en doute, etc.), de supposition (admettre, imaginer, supposer, à condition que, pourvu que, etc.), de possibilité (il est possible, il se peut), d'anticipation et de crainte (attendre, avoir peur, craindre, redouter, avant que, de crainte que, jusqu'à ce que, pour que, etc.), ainsi que dans les principales avec la particule que en position initiale, impliquant la variété de significations spécifiques : volonté, hypothèse, concession (voir à ce sujet Soutet $2000: 43-48)$.

\title{
2.2 L'opposition indicatif/subjonctif et l'exposition de faits
}

Il y a, en effet, des cas assez fréquents où l'implication de la (non-)prise en charge d'un événement par le locuteur n'explique pas l'emploi du subjonctif et de l'indicatif. Il nous suffit d'analyser l'exemple en (12) où l'emploi du subjonctif, introduit par ne pas douter, diffère de ceux que l'on a vus dans les exemples précédents. Ici, le personnage du curé d'un village prend en charge le contenu de la subordonnée, mais l'apparition du subjonctif fait ressortir la collision de deux événements, identifiés de façon implicite, en l'occurrence de deux opinions contraires, dignes d'être prises en considération, notamment, son opinion et celle du maire Voiturier, son adversaire principal dans le village. 
(12) Il [le curé] exposa rapidement l'affaire. [...] Certes, on aurait pu prétendre, et Voiturier n'y manqua pas, qu'il s'agissait d'un accident banal, ces amas de pierrailles étant fréquemment des nids de serpents. Mais comme personne n'avait été piqué depuis très longtemps, à l'exception de Beuillat et son cas était particulier, on ne douta pas que la Vouivre fût responsable. (Aymé 1972 : 239-240)

Force est bien de reconnaître que l'opposition entre le subjonctif et l'indicatif peut représenter une façon d'exposer des faits. Tandis que l'indicatif constate simplement un fait, le subjonctif en présente un, en impliquant un fait contraire qui, pour une raison quelconque mérite d'être pris en considération. C'est surtout évident dans l'exemple en (13), où le subjonctif est introduit par la construction il est exact que, majoritairement suivi de l'indicatif. Ici, l'emploi du subjonctif implique la possibilité d'une erreur que le personnage d'Angel suppose en constatant un fait.

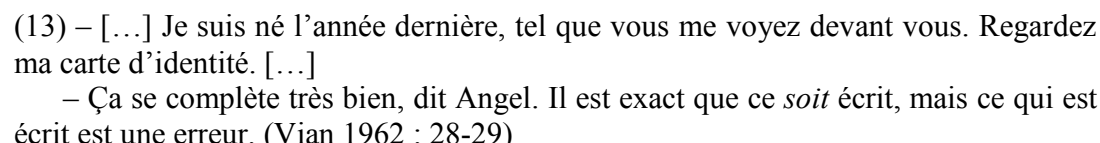

Afin d'offrir une illustration de la signification que l'opposition entre le subjonctif et l'indicatif sert à communiquer, comparons les exemples en (14)-(15) ci-dessous de l'emploi des deux modes avec la tournure concessive tout ... que. Globalement, la concession met en évidence sinon une opposition, du moins un décalage entre les espérances du locuteur, basées sur son expérience vécue, et un fait observé. Citons Leeman-Bouix (1994) à ce propos :

Donc la conjonction [bien que] suppose bien une prise en considération de deux possibilités [contraires], puisqu'elle introduit celle à laquelle on ne s'attend pas, par opposition (implicite) à celle à laquelle on s'attend. (Leeman-Bouix 1994: 92)

A notre avis, le subjonctif est envisageable dans un tel contexte lorsque le locuteur a l'intention de faire ressortir ce décalage, tandis que l'indicatif est employé là où ce décalage peut être négligé et même rejeté. Cette remarque vaut notamment pour le couple des exemples en (14)-(15) ci-dessous. Leur comparaison fait immédiatement apparaître que tant en (14) qu'en (15) les personnages ne laissent prise à aucun doute sur les contenus des subordonnées. Pourtant, en (14), l'emploi du subjonctif implique que le personnage de Mason met en relief la différence entre les marins britanniques (même s'ils sont des bandits ${ }^{4}$ ) et les Tahitiens « sauvages ».

(14) Vous avez bien dit: Voter !... Et comme si cela ne suffisait pas, vous voulez qu'à côté de ces hommes qui, tout bandits qu'ils soient, sont quand même des Britanniques, vous voulez que des Noirs, je dis bien : des Noirs, soient admis à siéger. (Merle 1962: 196)

L'emploi de l'indicatif dans l'exemple en (15), à l'inverse, met en relief l'absence de cette différence, en l'espèce les privilèges que Mason pourrait jouir en tant qu'officier.

(15) Si Mason préfère rester en cale sèche au lieu d'tirer un bord jusqu'ici, c'est son affaire. Mais la loi, c'est la loi, même pour Mason, tout officier qu'il est! (Merle $1962: 206)$

Une autre façon d'exposer des faits avec la prise en considération d'un événement contraire, c'est de les commenter, c'est-à-dire d'exprimer un jugement de valeur à leur propos au lieu de les constater. C'est parce qu'un jugement de valeur représente un sentiment ou une opinion personnelle du locuteur qui se forme d'une comparaison implicite de l'événement en question avec un événement contraire possible qui vaut d'être pris en compte. Comme le remarque Leeman-Bouix (1994) :

[...] pour pouvoir affirmer Je me réjouis, il faut prendre en compte les deux possibilités : ce qui me permet de définir mon sentiment si tu me comprends, c'est de peser celui que m'aurait causé ton incompréhension. Le subjonctif correspond donc bien ici, malgré les apparences, à la définition que l'on s'est donnée : le fait que tu me comprennes est mentalement mis en parallèle avec le fait que tu ne me comprennes pas, et c'est la comparaison de l'effet produit sur moi des deux comportements qui me 
permet de dire Je me réjouis que tu me comprennes (ou aussi bien Je suis désolé que tu ne me comprennes pas). (Leeman-Bouix 1994 : 91)

C'est pourquoi, le français standard requiert l'emploi du subjonctif dans un tel contexte linguistique. Pourtant, il y a des cas, si peu nombreux qu'ils soient, où l'indicatif s'impose mettant en évidence le résultat d'un événement sans prise en considération de possibilité contraire. C'est notamment le cas de l'exemple en (16) où le personnage de Mac Leod, le leader des marins mutins, est sûr que le résultat d'un vote sera défavorable pour un marin ce qui provoquera le mécontentement de celui-ci. C'est l'absence de toute autre possibilité que Mac Leod fait ressortir par l'emploi de l'indicatif dans cet exemple.

(16) Et y aura p'têtre un matelot qui sera pas bien content qu'le vent $a$ pas soufflé dans ses voiles. Dans c'cas, j'dis : fils, la loi, c'est la loi. (Merle 1962 : 206)

Outre les emplois du subjonctif que nous venons de citer, la prise en considération d'un événement contraire explique son usage dans des subordonnées, introduites par la conjonction après que. Il est bien connu que l'indicatif y est toujours largement attesté lorsqu'il s'agit de mettre en relief un événement qui est perçu comme un fait affirmé ou espéré et dont le contraire est vu comme improbable par le locuteur. En revanche, on a recours au subjonctif lorsqu'il s'agit d'impliquer une confrontation ou un décalage entre un fait actuel et ce que l'on avait supposé ou espéré pour réorienter l'attention de l'interlocuteur d'un résultat donné à la possibilité d'un résultat contraire qui mérite d'être pris en considération pour diverses raisons. Cette réflexion est illustrée par l'exemple en (17). On en conclut que le narrateur a eu des rapports sexuels avec une jeune fille nommée Jean, mais le subjonctif, employé dans cet exemple par la sœur de celle-ci, implique qu'il n'aurait pas dû le faire, mettant en évidence une possibilité contraire.

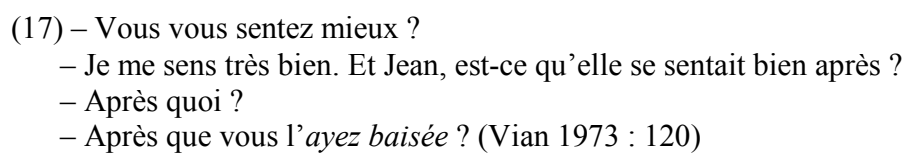

La possibilité d'un événement contraire s'observe aussi dans l'emploi du subjonctif dans des subordonnées, introduites par les conjonctions bien que, ce n'est pas que, sans que, etc., les expressions le fait que, comment se fait-il que, etc. et les verbes comprendre, concevoir, démentir, ignorer, nier qui désignent un fait réel, mais impliquent un décalage entre ce fait et un fait contraire, digne d'être pris en considération.

Ceux qui s'inspirent d'une approche polysémique pourraient objecter que le choix de mode après les verbes est motivé par leurs différentes valeurs (voir Soutet 2000 : 61-62). À cela on peut répliquer que si les valeurs des unités linguistiques étaient polysémiques, cela créerait un fardeau communicatif sur les interlocuteurs qui devraient retenir le cumul de toutes les idées, impliquées par les unités en question (tels les articles de dictionnaire), afin de rendre possible leur reconnaissance dans le discours. Il nous semble plus judicieux que l'on considère une forme linguistique comme un signe saussurien dont la valeur unique est assez complexe et abstraite pour être saisie sans réflexion par le locuteur, mais "soustendant » néanmoins ses 'choix' linguistiques » (Soutet 2000 : 131) d'une part et, d'autre part, servant d'indice de significations discursives à l'interlocuteur. Citons Huot (1986) à ce propos :

Je ne pense pas $[\ldots]$ que la solution qui consiste $[\ldots]$ à distinguer un double sens $[\ldots]$ pour une même unité lexicale soit la façon la plus satisfaisante d'en expliquer les différences d'interprétation selon les contextes d'emploi. (Huot 1986: 83-84, note 2)

\section{Conclusion}

Notre étude, basée à la fois sur la théorie du signe linguistique de Saussure et sur l'approche communicative de Martinet, a visé à présenter une hypothèse unitaire sur l'opposition entre l'indicatif et le subjonctif en français et surtout à expliquer les emplois exceptionnels des deux modes. Dans ce but, nous avons postulé des valeurs communes à tous les emplois de l'indicatif et du subjonctif, respectivement. Tandis que la valeur un événement de l'indicatif désigne un état, un devenir ou une action qui ont une réalité constatée ou très probable, la valeur un événement contraire considéré du subjonctif 
désigne la prise en considération implicite d'un événement contraire qui pourrait se produire contrairement aux prévisions du locuteur. Les exemples attestés, dont la plupart sont exceptionnels, ont fait montrer que cette opposition permet de justifier la totalité des emplois des deux modes, réfutant ainsi une objection possible que l'explication proposée est trop simplificatrice et donc fausse. Nous avons réparti ces emplois en deux grands groupes en conformité avec leurs buts communicatifs. L'opposition entre le subjonctif et l'indicatif, nous l'avons vu, est employée soit pour marquer le degré de la prise en charge d'un événement par le locuteur, soit pour exposer des faits avec ou sans prise en considération d'un événement contraire, respectivement.

Et pourtant, il ne faut point conclure de ce qui précède que l'emploi des modes soit imprévisible. Le locuteur français pourrait employer le subjonctif partout, avec une légère restriction, pour certains buts communicatifs. S'il n'est pas toujours libre dans son choix, c'est parce que, comme nous l'avons souligné plus haut, la tendance de toutes les langues est de réduire le nombre d'irrégularités et de les systématiser toutes dans la mesure du possible. Dans ces conditions, une analyse textuelle représente une contribution méthodologique importante. En effet, si les valeurs proposées sont correctes et qu'elles justifient l'apparition des deux modes dans les exemples individuels, on peut prévoir que leur emploi dans les textes littéraires ne sera pas aléatoire non plus. C'est parce que chaque texte peut être considéré comme un inventaire de différentes idées (contextes, thèmes ou leitmotivs), organisées autour d'une idée maîtresse. Aussi, proposons-nous, à l'étape suivante, d'analyser la distribution de l'indicatif et du subjonctif à travers des textes littéraires. La thèse que nous défendons ici, suivant Tobin (1990, 1993, 1995), est que dans chaque texte il y a des contextes qu'un mode peut servir à caractériser, mieux que tout autre mode, en raison de sa valeur linguistique. En d'autres termes, le choix de l'un ou de l'autre mode peut être attribué à des thèmes particuliers, des personnages spécifiques et des enchaînements d'événements, constituant le sujet principal ou les actions secondaires d'un texte. Si dans des contextes particuliers l'emploi de tel ou tel mode est régulier et réitéré, cela créera une sensation de cohérence et de cohésion textuelles et, par conséquent, montrera que les hypothèses suggérées ne sont pas gratuites.

\section{Références bibliographiques}

Benveniste, É. (1966). Problèmes de linguistique générale. Vol. 1. Paris : Gallimard.

Börjeson, L. (1966). La fréquence du subjonctif dans les subordonnées complétives introduites par «que » étudiée dans des textes français contemporains. Studia-Neophilologica, 38, 3-64.

Boysen, G. (1971). Subjonctif et hiérarchie : étude sur l'emploi du subjonctif dans les propositions complétives objets de verbes en français moderne. Odense : Odense University Press.

Clairis, Ch. (2005). Vers une linguistique inachevée. Louvain : Peeters.

Cohen, M. (1965). Le subjonctif en français contemporain. Paris : Société d'édition d'enseignement supérieur.

Dreer, I. (2007). Expressing the Same by the Different: the Subjunctive versus the Indicative in French. Amsterdam: John Benjamins.

Ducrot, O. (1972). Dire et ne pas dire. Paris : Hermann.

Fleischman, S. (1982). The future in thought and language: diachronic evidence from Romance. Cambridge: Cambridge University Press.

Gosselin, L. (2010). Les modalités en français : la validation des représentations. Amsterdam : Rodopi.

Grevisse, M. (2001). Le bon usage : grammaire française. $13^{\mathrm{e}}$ édition, sous la direction d'André Goosse. Paris : Éditions Duculot.

Guillaume, G. (1970). Temps et verbe : théorie des aspects, des modes et des temps; suivi de l'architectonique du temps dans les langues classiques. Paris : Honoré Champion.

Huot, H. (1986). Le subjonctif dans les complétives : subjectivité et modalisation. In Mitsou, R.; Couquaux, D. (éds), La grammaire modulaire, Paris : Éditions de Minuit, 81-111. 
Jonge, B. de. (2001). Spanish subjunctive mood: one form, more than one meaning? In Bok-Bennema, R.; Jonge B. de; Kampers-Manhe, B.; Molendijk, A. (éds), Faux titre: adverbial modification, Amsterdam: Rodopi, 79-93.

Kirsner, R. S. (2002). The Future of a Minimalist Linguistics in a Maximalist World. In Reid, W.; Otheguy, R.; Stern, N. (éds), Signal, Meaning, and Message: Perspectives on Sign-based Linguistics, Amsterdam: John Benjamins, 339-371.

Kirsner, R. S. (2004). Introduction: on paradigms, analyses, and dialogue. In Contini-Morava, E.; Kirsner, R. S.; Rodríguez-Bachiller, B. (éds), Cognitive and communicative approaches to linguistic analysis, Amsterdam: John Benjamins, 1-21.

Lagerqvist, H. (2009). Le subjonctif en français moderne : esquisse d'une théorie modale fondée sur des textes non littéraires. Paris : Presses de l'Université Paris-Sorbonne.

Lalaire, L. (1998). La variation modale dans les subordonnées à temps fini du français moderne: approche syntaxique. Bern : Peter Lang.

Leeman-Bouix, D. (1994). Grammaire du verbe français : des formes au sens. Modes, aspects, temps, auxiliaires. Paris : Fernand Nathan.

Martin, R. (1983). Pour une logique de sens. Paris : Presses universitaires de France.

Martin, R. (1987). Language et croyance. Les «univers de croyance» dans la théorie sémantique. Bruxelles : Pierre Mardaga.

Martinet, A. (1973). Pour une linguistique des langues. Foundations of Language, Volume 10, 339-369. Dordrecht, The Netherlands : D. Reidel.

Moignet, G. (1959). Essai sur le mode subjonctif en latin postclassique et en ancien français. Paris : Presses universitaires de France.

Nølke, H. (1993). Le regard du locuteur. Pour une linguistique des traces énonciatives. Paris : Éditions Kimé.

Nølke, H. (2001). Le regard du locuteur. Pour une linguistique des traces énonciatives. Vol. 2. Paris : Éditions Kimé.

Nordahl, H. (1969). Les systèmes du subjonctif corrélatif. Étude sur l'emploi des modes dans la subordonnée complétive en français moderne. Bergen : Universitetsforlaget.

Poplack, S. (1992). The inherent variability of the French subjunctive. In Laeufer, Ch.; Morgan, T. A. (éds), Theoretical analyses in Romance linguistics, Amsterdam : John Benjamins, 235-263.

Reid, W.; Gildin, B. (1979). Semantic Analysis without the Sentence. In Clyne, P.; Hanks, W.; Hofbauer, C. (eds), The Elements: A Parasession in Linguistic Units and Levels. Chicago Linguistic Society, 163-174.

Saussure, F. de. (2001 [1916]). Cours de linguistique générale. Sous la dir. de Charles Bally et Albert Sechehaye avec la collaboration de Albert Riedlinger. Paris : Éditions Payot \& Rivages.

Soutet, O. (2000). Le subjonctif en français. Paris : Ophrys.

Stefanini, J. (1992). Linguistique et langue française. Paris: Éditions du Centre National de la Recherche Scientifique.

Tobin, Y. (1990). Semiotics and linguistics. London: Longman.

Tobin, Y. (1993). Aspect in the English verb: process and result in language. London: Longman.

Tobin, Y. (1995). Invariance, markedness and distinctive feature analysis: a contrastive study of sign systems in English and Hebrew. Amsterdam: John Benjamins.

Togeby, K. (1982). Grammaire française. Les formes personnelles du verbe. Volume II. Copenhague : Akademisk Forlag.

Touratier, Ch. (1996). Le système verbal français. (Description morphologique et morphématique). Paris : Masson \& Armand Colin

\section{Sources}

Aymé, M. (1972). La Vouivre. Paris : Gallimard. 
Beauvoir, S. de. (1943). L'invitée. Paris : Gallimard.

Camus, A. (1947). La peste. Paris : Gallimard.

Le Clézio, J.-M. G. (1978). Mondo et autres histoires. Paris : Gallimard.

Libération. 30-31 octobre 1999.

Merle, R. (1962). L'île. Paris : Gallimard.

Montherlant, H. de. (1954). Les olympiques. Paris : Gallimard.

Sagan, F. (1962). Les violons parfois. Paris : Éditions Julliard.

Vian, B. (1962). L'arrache-cour. Paris : Éditions Jean-Jacques Pauvert.

Vian, B. (1973). J'irai cracher sur vos tombes. Paris : France loisirs.

\footnotetext{
${ }^{1}$ Pour la présentation plus détaillée et critique des théories linguistiques pour le subjonctif français, nous renvoyons à Moignet (1959), Dreer (2007) et Soutet (2000).

${ }^{2}$ Pour d'autres objections auxquelles nous avons déjà répondu, voir Dreer (2007:96).

${ }^{3}$ Il faut rappeler qu'à la fin du livre Rieux reçoit un télégramme, lui annonçant la mort de sa femme.

${ }^{4}$ La tradition veut que le subjonctif soit exclu après $s i$, y compris la tournure concessive même si. Il sort de notre propos d'entrer dans les détails et d'examiner de plus près cette exclusion. Bornons-nous ici à observer que le $s i$ introduit la possibilité au sens le plus général du mot (à comparer avec le que qui peut introduire seulement une possibilité spécifique).
} 